more severe effects on neurodevelopment than deletion (i.e. loss of genetic material), whereas at other loci the opposite may be the case.

Notwithstanding our differences, we share with Dr Crespi a desire to develop new theoretical frameworks within which to view the increasing volume of novel genetic insights coming from genetics. ${ }^{5}$ This will allow us to test specific biological hypotheses that can advance understanding of psychiatric illness in general and the relationship between schizophrenia and autism in particular. This will help move psychiatric classification from descriptive traditions based upon expert opinion towards robust empirical evidence that more closely relates to the workings of the brain.

1 Craddock N, Owen MJ. The Kraepelinian dichotomy - going, going . . . but still not gone. Br J Psychiatry 2010; 196: 92-5.

2 Rzhetsky A, Wajngurt D, Park N, Zheng T. Probing genetic overlap among complex human phenotypes. Proc Natl Acad Sci USA 2007; 104: 11694-9.

3 Larsson HJ, Eaton WW, Madsen KM, Vestergaard M, Olesen AV, Agerbo E, et al. Risk factors for autism: perinatal factors, parental psychiatric history, and socioeconomic status. Am J Epidemiol 2005; 161: 916-25.

4 Kirov G, Rujescu D, Ingason A, Collier DA, O'Donovan MC, Owen MJ. Neurexin 1 (NRXN1) deletions in schizophrenia. Schizophr Bull 2009; 35 : 851-4.

5 Cross-Disorder Phenotype Group of the Psychiatric GWAS Consortium. Dissecting the phenotype in genome-wide association studies of psychiatric illness. Br J Psychiatry 2009; 195: 97-9.

Nick Craddock, Michael J. Owen, MRC Centre for Neuropsychiatric Genetics and Genomics, School of Medicine, Cardiff University, Heath Park, Cardiff CF14 4XN, UK. Email: craddockn@cardiff.ac.uk

doi: 10.1192/bjp.196.6.495a

\section{'Stoned': Schneiderian first-rank symptoms as a manifestation of bilateral obstructive urolithiasis}

In October 2009, a 22-year-old student living with his parents requested treatment in our emergency ward. The patient seemed very troubled. During psychiatric evaluation, he reported that for 10 days he had been hearing voices that had commented on his actions and had commanded him not to think specific thoughts. He also felt that some of his thoughts had been introduced into his brain. The voices had discussed that people in his environment were controlled by computer software. Furthermore, the patient reported to have the impression that his body parts had been manipulated in size, and that he had been exhausted during the past week, suffering from episodes of mild fever. The degree of psychiatric symptoms had been increasing slowly over time. His general practitioner, who had only been informed about the general feeling of illness and episodes of fever, had suspected tonsillitis and treated the patient with methyl penicillin for 7 days. Because the somatic symptoms had not improved satisfactorily, treatment had been switched to azithromycin. The patient further reported a history of cannabis use and abstinence of 3 years. He had never before experienced psychotic symptoms, and none of his first-degree relatives had been diagnosed with psychiatric illnesses.

The patient was well oriented and awake. There were no pathological findings in the general examination and no focal neurological signs. A cranial computed tomography scan was without pathological findings, and analysis of the cerebrospinal fluid showed normal values. Laboratory testing revealed high levels of leucocytes $(8.9 / \mathrm{nl})$, c-reactive protein $(203.7 \mathrm{mg} / \mathrm{l})$ and procalcitonin $(7.03 \mathrm{ng} / \mathrm{ml})$ as well as of serum creatinine $(3.32 \mathrm{mg} / \mathrm{dl})$ and urea $(90 \mathrm{mg} / \mathrm{dl})$. While diagnostics were performed, the patient developed fever with an axillary temperature of $39^{\circ} \mathrm{C}$. Ultrasound investigation showed primary bilateral hydronephrosis and a native low-dose computerised tomography scan of the abdomen revealed bilateral urinary stones, causing urinary retention, renal failure and finally urosepsis.

After admission to the intermediate care unit, the patient was treated with intravenous paracetamol and meropenem, but he refused the recommended psychopharmacological medication. Urinary retention was managed via bilateral placement of J-stents. The left prevesical stone was completely extracted via ureteroscopy on day 13 without complications. The right stone was extracted in a second intervention. Further testing revealed no detectable causes for urolithiasis, including normal values for calcium and parathyroid hormone. After relief of obstruction and at 2 weeks followup, the psychopathological symptoms were fully remitted without antipsychotic treatment.

In summary, the following clinical course has to be assumed: bilateral urolithiasis with urinary retention had led to a urinary tract infection with intermittent episodes of mild fever, but without noticeable oliguria or severe disability. The patient further developed a first-time acute psychotic episode, displaying firstrank symptoms of schizophrenia in absence of clouding of consciousness or lowering of vigilance (i.e. without the presence of a delirium according to DSM-IV criteria ${ }^{1}$ ). Although the relation of intermittent fever and psychotic symptoms cannot be reconstructed, symptoms were present at the time of examination in the emergency ward without febrile temperature. Laboratory testing revealed that the patient had finally developed a systemic inflammatory response syndrome and presented with urosepsis requiring emergency treatment.

Despite laboratory signs of post-renal acute renal failure, there was no clinically significant uremia at presentation. Although there are well-known reports on the psychiatric aspects of uremia ${ }^{2}$ and paranoid psychoses with uremia, ${ }^{3}$ these mechanisms are not sufficiently able to explain the presented case. Furthermore, although a syndrome with anxiety, panic and hallucinations (Hoignés syndrome) can be induced by therapy with procaine penicillin or amoxicillin, ${ }^{4}$ the onset of psychotic symptoms preceded the penicillin regimen in our patient, rendering a causal role of antibiotic treatment improbable.

Thus, this case represents the first published report on urolithiasis with secondary urosepsis presenting with Schneiderian first-rank symptoms. It exemplifies the broad phenomenology of organic psychoses and once more illustrates that profound somatic diagnostics are mandatory during the evaluation of a first psychotic episode. Additionally, it provides further evidence for the non-specificity of first-rank symptoms for schizophrenia as it is currently discussed with respect to the development of DSM-V. ${ }^{5}$

\section{Acknowledgements}

We thank the patient for giving consent to publication of this report. J.H. and C.G.H contributed equally to this report.

1 American Psychiatric Association. Diagnostic and Statistical Manual of Mental Disorders (4th edn) (DSM-IV). APA, 1994. 
2 Baker AB, Knutson J. Psychiatric aspects of uremia. Am J Psychiatry 1946; 102: 683-7.

3 Menninger KA. Paranoid psychosis with uremia. J Nerv Ment Disord 1924; 60 : 26-34.

4 Beal DM, Hudson B, Zaiac M. Amoxacillin-induced psychosis? Am J Psychiatry 1986; 143: 255-6.

5 Nordgaard J, Arnfred SM, Handest P, Parnas J. The diagnostic status of firstrank symptoms. Schizophr Bull 2008; 34: 137-54.
Johannes Huber, Department of Urology, University of Heidelberg, Germany; Christian G. Huber, Department of Psychiatry and Psychotherapy, University

Medical Centre Hamburg-Eppendorf, Martinistr. 52, D-20246 Hamburg, Germany.

Email: c.huber@uke.uni-hamburg.de; Martin Lambert, Department of Psychiatry and

Psychotherapy, University Medical Centre Hamburg-Eppendorf, Hamburg; Markus

Hohenfellner, Department of Urology, University of Heidelberg, Germany

doi: 10.1192/bjp.196.6.496

\section{Correction}

Early intervention in panic: pragmatic randomised controlled trial. BJP, 196, 326-331. Table 1 (p. 329): the ranges given for PDSS-SR, HADS-Anxiety, Mobility Inventory and BID-II are the theoretical ranges of these scales.

doi: 10.1192/bjp.196.6.497 